\title{
A nuclear data approach for the Hubble constant measurements
}

\author{
Boris Pritychenko ${ }^{\mathrm{a}}$ \\ National Nuclear Data Center, Brookhaven National Laboratory, Upton, NY 11973-5000, USA
}

\begin{abstract}
An extraordinary number of Hubble constant measurements challenges physicists with selection of the best numerical value. The standard U.S. Nuclear Data Program (USNDP) codes and procedures have been applied to resolve this issue. The nuclear data approach has produced the most probable or recommended Hubble constant value of 67.2(69) $(\mathrm{km} / \mathrm{sec}) / \mathrm{Mpc}$. This recommended value is based on the last 20 years of experimental research and includes contributions from different types of measurements. The present result implies $(14.55 \pm 1.51) \times 10^{9}$ years as a rough estimate for the age of the Universe. The complete list of recommended results is given and possible implications are discussed.
\end{abstract}

\section{Introduction}

Hubble's law and the constant play an important role in modern astrophysics and cosmology [1,2]:

$$
v=H_{o} \cdot D
$$

where are $v$ is the recessional velocity $(\mathrm{km} / \mathrm{sec}), H_{0}$ is Hubble's constant and $D$ is the proper distance from the galaxy to the observer. For many years, researchers have been improving the accuracy of the constant [3]. The large number of measurements [4] creates a certain degree of confusion about Hubble constant numerical value, and scientists often rely on recently-published results. The precision of Hubble constant measurements has improved enormously over the years; however, it is not always prudent to reject older results in favor of the latest findings. Consequently, it makes perfect sense to analyze all available results, evaluate the data, and extract the recommended value.

\section{Hubble constant evaluation}

The volume of Hubble constant measurements far exceeds other experimental quantities in physics [5]. Previously, similar situations have been encountered in nuclear and particle physics and resolved with data evaluations. Nuclear data evaluations and their policies are well described in literature [6,7]. Frequently, the evaluations are completely based on or adjusted to available experimental data. These adjustments and specialized mathematical statistics techniques can be applied for nuclear, particle, or any other data sets.

In this work, I would follow standard nuclear data evaluation procedures to deduce the recommended value. Current evaluation input data are mostly based on the NASA/HST Key Project on the Extragalactic Distance Scale compilation [4] and recent results. A study of historical Hubble Constant measurements [4] is instrumental in the data analysis. It suggests that one may safely reject all measurements prior to 1970 . It is common

a e-mail: pritychenko@bnl.gov knowledge that Hubble constant measurements heavily rely on the accuracy of astronomic distance determination. Older results, such as those reported by A. Sandage and G. de Vancouleurs, Hubble telescope maintenance (1990-1996), etc., suffered from inaccurate measurements [3]. Therefore, the rejection of all results prior to 1997 could provide a complimentary benchmark value of the Hubble constant.

In the present data analysis, the experimental data have been separated into two groups, with 1970 and 1997 time cuts, and further reduced using the following policies:

- Rejection of repeated results (multiple publication of the same result)

- Rejection of model-dependent results

- Rejection of potential outliers using Chauvenet's criterion [8].

Common data evaluation practices indicate that recommended value should be based on a large statistical sample that includes different types of measurements. The 1970 and 1997 redacted data sets of $\sim 334$ and $\sim 218$ data points, respectively, provide such samples. These large samples create the possibility of deducing Hubble constant value for each method of observation besides the combined value that is based on all measurements. The current data collections were further subdivided using a NASA/HST Key Project on the Extragalactic Distance Scale classification of experimental methods [4]:

- $\mathrm{S}=$ Type Ia supernovae (SNIa)

- 2 = Type II supernovae (SNII)

- $\mathrm{L}=$ Lens

- $r=$ Red Giants

- $\mathrm{B}=$ Baryonic Tully-Fisher

- $\mathrm{R}=$ Inverse Tully-Fisher (ITF,RTF)

- $\mathrm{H}=$ Infrared Tully-Fisher (or IRTF)

- $\mathrm{F}=$ Fluctuations

- $\mathrm{A}=$ Global Summary

- $\mathrm{Z}=$ Sunyaev-Zeldovich

- $\mathrm{T}=$ Tully-Fisher

(c) The Authors, published by EDP Sciences. This is an Open Access article distributed under the terms of the Creative Commons Attribution License 4.0 (http://creativecommons.org/licenses/by/4.0/). 


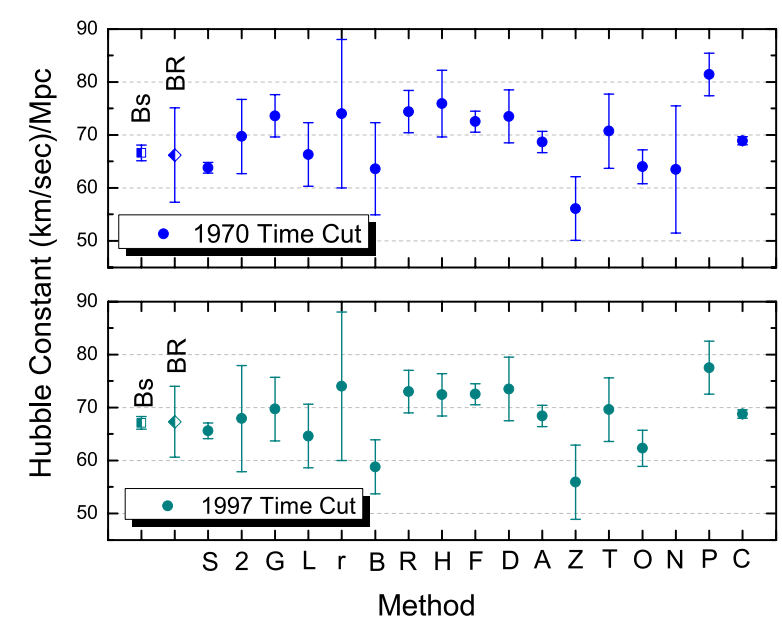

Figure 1. Evaluated values of Hubble constant using 1970 and 1997 time cuts for experimental data. These plots include the evaluated or recommended values for combined observations, using the Best Representation (BR) and Bootstrap (Bs) data analysis techniques. Globular cluster luminosity function $(\mathrm{G})$, Dn-Sig/Fund Plane (D), Novae (N), Planetary nebula luminosity function $(\mathrm{P})$ and $\mathrm{CMB}$ Fit $(\mathrm{C})$ values are also shown.

$$
\text { - } \mathrm{O}=\text { Other }
$$

These experimental data sets have been processed with the latest version of the visual averaging library [8]. The library program includes limitation of relative statistical weight (LWM), normalized residual (NRM), Rajeval technique (RT), and the Expected Value (EVM) statistical methods to calculate averages of experimental data with uncertainties. The experimental data sets were processed, and evaluated values with reduced $\chi^{2}<2$ were typically accepted as reasonable data fits. The current evaluation incorporates statistical methods based on the inverse squared value of the quoted uncertainties, a procedure that is consistent with the general methodology used in treatment of data for the ENSDF database [7] and Particle Data Group [9].

\section{Results and discussion}

Two sets of recommended values are displayed in Fig. 1, and the combined numerical values are shown in Table 1. The Hubble constant combined central values extracted by means of different mathematical techniques are in good agreement, while uncertainties need further discussion. Visual inspection of the numerical values shown in the Fig. 1 indicates the Best Representation mathematical procedure (depicted as "BR") provides a best fit for different experimental methods with reasonable uncertainties. Other procedures such as the Bootstrap technique (depicted as "Bs") result in rather small uncertainties. These small uncertainties are due to specifics of data analysis procedures such as assignment of different statistical weights to results with different uncertainties. In light of this disclosure, it is prudent to select the Best Representation results as the recommended value.

Finally, two different time cuts of 1970 and 1997 for Hubble's data have yielded two recommended values of 66.2(89) and 67.2(69) $(\mathrm{km} / \mathrm{sec}) / \mathrm{Mpc}$, respectively. The agreement between these values partially reflects the fact
Table 1. Results of the Hubble Constant evaluation for all observations using 1970 and 1997 time cuts.

\begin{tabular}{c|c|c}
\hline \hline Method & $\begin{array}{c}\text { Time Cut: 1970 } \\
(\mathrm{km} / \mathrm{sec}) / \mathrm{Mpc}\end{array}$ & $\begin{array}{c}\text { Time Cut: 1997 } \\
(\mathrm{km} / \mathrm{sec}) / \mathrm{Mpc}\end{array}$ \\
\hline Unweighted Average & $67.56(73)$ & $66.77(100)$ \\
Weighted Average & $63.68(58)$ & $66.54(100)$ \\
LWM & $67.30(93)$ & $66.54(100)$ \\
Normalized Residual & $64.50(50)$ & $65.85(100)$ \\
Rajeval Technique & $65.57(45)$ & $66.40(100)$ \\
Best Representation (BR) & $66.2(89)$ & $67.2(69)$ \\
Bootstrap (BS) & $66.6(15)$ & $67(13)$ \\
Mandel-Paule & $66.7(92)$ & $66.5(49)$ \\
\hline \hline
\end{tabular}

that the majority of the Hubble's constant measurements has been performed in the last 20 years, and a small number of potential outliers has been rejected. More accurate recent observations imply a preference for the 1997 time cut value of $67.2(69)(\mathrm{km} / \mathrm{sec}) / \mathrm{Mpc}$. The last result is consistent with the Hubble Space Telescope and Wide Field Camera 3 [10], and agrees well with the Planck's Mission and WMAP values [9,11] and a scale factor $0.678(9)$ for the Hubble expansion rate of $100(\mathrm{~km} / \mathrm{sec}) / \mathrm{Mpc}$ [9]. Inclusion of the globular cluster luminosity function, Dn-Sig/Fund Plane, Novae and planetary nebula luminosity function data would slightly change 1970 and 1997 recommended values to 66.9(90) and 67.5(69), respectively. These less precise measurements do not affect the recommended values severely because of a Chauvenet's criterion analysis.

In recent years, an effort has been made to calculate the Hubble constant median statistics [12]. The median statistics approach differs substantially from the nuclear or particle data evaluation procedures. At the same time, it provides a complementary value of $68 \pm 5.5$ (or \pm 1 ) $(\mathrm{km} / \mathrm{sec}) / \mathrm{Mpc}$ that can be compared with the present results.

The good knowledge of Hubble constant value has multiple implications in science. As an example, a rough estimate of the age of the Universe can be deduced using the standard methodic [11]. The adopted value of $67.2(69)(\mathrm{km} / \mathrm{sec}) / \mathrm{Mpc}$ implies $(14.55 \pm 1.51) \times 10^{9}$ years estimated value for the age of Universe. The last result is consistent with the recently published value of $(13.798 \pm 0.037) \times 10^{9}$ years [13].

\section{Conclusions}

The analysis of Hubble constant measurements has been performed using standard USNDP codes and procedures. An evaluated data set of most probable values of Hubble constant has been deduced and shown in the Table 1 . These values are consistent with other available results. An accurate constant value is instrumental for many potential applications. The recommended value of the constant is completely based on experimental measurements, and further, more precise observations, would lead to more accurate determination of it.

The author is indebted to Dr. M. Herman (BNL) for support of this project and grateful to Dr. V. Unferth (Viterbo University) for help with the manuscript. This work was funded by the Office of Nuclear Physics, Office of Science of the U.S. Department 
of Energy, under Contract No. DE-AC02-98CH10886 with Brookhaven Science Associates, LLC.

\section{References}

[1] R.N. Boyd, An Introduction to Nuclear Astrophysics. The University of Chicago Press, Chicago and London (2008). ISBN-10: 0-226-06971-0, ISBN-13: 978-0-226-06971-5

[2] A.D. Dolgov, Ya. B. Zel'dovich, M.V. Sazhin, Cosmology of the Early Universe. The University of Moscow Press, Moskva (1988). In Russian. ISBN 5-211-00108-7

[3] M. Livio, A.G. Riess, Physics Today, October 2013, 41 (2013)

[4] J. Huchra, The NASA/HST Key Project on the Extragalactic Distance Scale, https://www. cfa.harvard.edu/ dfabricant/huchra/ hubble.plot.dat; downloaded May 19, 2015
[5] B. Pritychenko, J. Phys. G: Nucl. Part. Phys. 42, 075103 (2015)

[6] B. Pritychenko, J. Choquette, M. Horoi, B. Karamy, B. Singh, At. Data Nucl. Data Tables 98, 798 (2012)

[7] Evaluated Nuclear Structure Data Files (ENSDF), http://www.nndc.bnl.gov/ensdf, downloaded May 24, 2015; T.W. Burrows, Nucl. Instr. and Meth. A 286, 595 (1990)

[8] M. Birch, B. Singh, Nuclear Data Sheets 120, 106 (2014)

[9] C. Patrignani, et al. (Particle Data Group), Chin. Phys. C 40, 100001 (2016)

[10] A.G. Riess, et al., arXiv:1103.2976 (2011)

[11] Hubble's law, http://en.wikipedia.org/ wiki/Hubble\%27s_law; downloaded May 19, 2015

[12] G. Chen, B. Ratra, arXiv:1105.5206 (2011)

[13] Planck Collaboration, A\&A 571, A1 (2014) 